\title{
QUANTUM FLUCTUATIONS IN CUBIC FERROMAGNETS
}

\author{
J. Sznajd, M. Dudziński \\ Institute of Low Temperature and Structure Research, \\ Polish Academy of Sciences, 50-950 Wroctaw, Poland
}

(Received 19 July 1999)

\begin{abstract}
The theory of phase transitions in quantum spin ferromagnets with cubic single ion anisotropy is reviewed. Some fundamental difficulties with the mean-field approach to these systems are discussed. It is pointed out that quantum fluctuations drive the quadrupolar long range order in cubic ferromagnets with three easy axes. This order cannot be observed in the classical counterpart of the considered model. A new decimation procedure to study magnetic chains with multi-spin interaction is proposed and applied to investigate effective Hamiltonian of the cubic ferromagnet with large anisotropy.
\end{abstract}

Key words: quantum fluctuations, quadrupolar order, cubic ferromagnet.

PACS numbers: 75.10.Jm, 75.10.Dg, 75.30.Gw

\section{INTRODUCTION}

It is of course well known that the origin of the Heisenberg exchange spin Hamiltonian is in quantum mechanics. However, in spite of the fact that the ferromagnetism cannot exist in the classical limit usually in the theory of phase transitions one treats spins, particularly $S \gg 1$, as classical vectors. It is widely believed that the finite temperature critical behaviour should not be affected by the quantum effects but the quantum nature of physical system cannot be ignored if one considers the ground state properties and the character of the low temperature phase. For both of these problems the quantum nature of the systems with higher spin values can be even more significant than the quantum nature of the systems with so-called the most quantum spin $S=1 / 2$. The importance of the quantum character of the spins for the ground state of the antiferromagnetic chains was pointed out by Haldane [1] who argued that there is a fundamental difference between integer and half-integer spin chains. The influence of the spin value on the existence of the various states has been studied by Zittartz and coworkers [2]. They found the exact ground states for a large class of spin-1 and $3 / 2$ models on low dimensional lattices and presented, between others, how rich structure the $T=0$ phase diagrams of the quantum spin systems with $S>1$ can show.

The significance of the quantum character of spins is not restricted to the ground state of the low dimensional antiferromagnets. It is surprising but quantum effects can affect for example also the phase transitions in the cubic ferromagnets on one-, two- or three- dimensional lattices and not only in the ground state. In the present paper we show that as a consequence of the quantum character of spins the finite temperature phase diagram of the cubic ferromagnet is essentially different for integer and half-integer spin $[3,4,5]$; the quadrupolar long range order can be observed in the paramagnetic ground state and in the paramagnetic low temperature phase of the $S=2$ system without a quadrupolar type of interaction (quantum fluctuation driven phase transi- tion) $[6,7,8]$. We also propose the real space renormalization group (RSRG) technique with linear weight operator (decimation procedure) to study the effective quantum Hamiltonian of the cubic ferromagnetic chain.

\section{THE MODEL}

The Hamiltonian we consider is that of a system with cubic single ion anisotropy

$$
H=-J \sum_{\langle i j\rangle}\left(\mathbf{S}_{i} \bullet \mathbf{S}_{j}\right)+\frac{1}{3} K \sum_{i, \alpha}\left(S_{i}^{\alpha}\right)^{4},
$$

where $S_{i}^{\alpha}(\alpha=x, y, z)$ is the $\alpha$ component of the spin operator for $S \geq 2(S=2$ is the lowest value for which the single ion term is non-trivial) and $K$ is a parameter associated with crystal field anisotropy of cubic symmetry. In this quantum ferromagnetic model $(J>0)$ the sign of $K$ determines whether the spins tend to align along the cubic axes $(K<0)$ - three easy axis, or along a main diagonal $(K>0)$ - four easy axes. The model (1) was studied within the framework of molecular field approximation (MFA) by several authors [3]. It was found in this very simple approximation that similarly to the antiferromagnetic chains there is an essential difference between the cubic ferromagnets with integer and half-integer spins. Within the MFA one obtains that for $K<K_{c}=-4 z J$ ( $z$ denotes the number of nearest neighbours) a system described by the Hamiltonian (1) with $S=2$ does not exhibit any magnetic long range order for an arbitrary temperature whereas the same model with $S=5 / 2$ should have magnetic long range order for any value of anisotropy at sufficiently low temperature. In this approximation it means that the models with $S=2$ (and other integer spins) have no long range order even in the ground state for $K<-4 z J$. In other words at finite temperatures for sufficiently large, negative, anisotropy constant $K$, in the models with integer spins, in opposite to the models with half-integer no phase transitions 
exist.

If one takes into account the critical fluctuations [5,9], the general scenario survives although these fluctuations can change the character of the phase transitions from continuous to discontinuous for some values of the anisotropy constant. In fact for the most interesting case, the ferromagnets with three easy axes, the character of the phase transition is still unsettled. The earlier results found by using momentum space renormalization group (RG) [9] suggested that this transition can be continuous or discontinuous in dependence on the values of the model parameters, however, recently on the basis of new calculations [10] it has been concluded [7] that the ferromagnets with three easy axes can undergo only a discontinuous phase transition.

In recent papers $[6,7,8]$ we have considered the influence of the quantum fluctuations on the ground state and low temperature behaviour of the cubic ferromagnets with three easy axes. The natural question is if there is some other kind of long range order (non-magnetic) in the cubic ferromagnets with large negative anisotropy omitted by the MFA - theory which neglects any fluctuations and RG - which takes into account only critical fluctuations.

In a system with higher value of spin $S \geq 1$ in addition to the magnetic (dipolar) long range order one can observe also multipolar order e.g. quadrupolar one. It is obvious that a magnetic ground state will always have quadrupolar moment while for a non-magnetic (paramagnetic) state this may be not true. Of course one can expect the existence of the quadrupolar ordering even without dipolar one if there is a quadrupolar type of interaction in the system under consideration. The question is if such an order can appear in the system with only magnetic interaction and single-ion anisotropy. It is clear that such a possibility does not exist for the classical spin systems. It is also easy to see that any kind of the MFA cannot lead to the multipolar ordering in the phase without magnetic long-range order. However, the quantum fluctuations can lead to qualitatively different physical properties.

Unfortunately, there is no plausible method which allows us to study efficiently the $S=2$ model even in the ground state. So, we have applied several complementary methods: perturbation theory, real space renormalization group (RSRG), MFA and density matrix renormalization group (DMRG) to show that in the cubic ferromagnets with three easy axes $(K<0)$ one can observe quantum fluctuation driven phase transition to the quadrupolar phase.

\section{PERTURBATION THEORY}

We have applied perturbation theory for $J /|K| \ll 1$, treating the exchange term in the Hamiltonian (1) as a perturbation. In the zeroth order approximation one has the set of independent spins with the crystal field interaction of the cubic symmetry. This field splits the five states of the $S=2$ spin into a triplet and a nonmagnetic doublet which for the considered case $K<0$ is favoured. In the standard way we have found the contributions to the effective Hamiltonians up to the fourth order for the systems in 1, 2 and 3 dimensions [6]. In the second order calculation the effective Hamiltonian has the same form in all dimensions

$$
H_{e f f}^{(2)}=\frac{3}{2} \frac{J^{2}}{K} \sum_{\langle i j\rangle}\left(\sigma_{i}^{x} \sigma_{j}^{x}+\sigma_{i}^{y} \sigma_{j}^{y}\right) .
$$

Thus, we have obtained an effective interaction between the nonmagnetic states of the doublet in the form of the $X Y$ model Hamiltonian. This is a hint that the MFA predictions about the state without long range order for large, negative, $K$ may be incorrect because the $X Y$ model is known to exhibit the long range order in $d=2$ and 3 [11], and to be critical in $d=1$ [12].

The Hamiltonian (2) has a continuous rotational symmetry in its $X Y$ plane, whereas the original model possesses a discrete cubic symmetry. This difference is of course the result of the approximation. The symmetry breaking terms are not present also in the third-order contribution to $H_{e f f}$, where additional interaction between the $z$-spin components appears

$$
-\frac{3 J^{3}}{4 K^{2}} \sum_{\langle i j\rangle} \sigma_{i}^{z} \sigma_{j}^{z}
$$

The symmetry breaking terms are found in the fourth order calculation and they have forms of three-spin interactions

$$
\begin{aligned}
H_{3 s}^{(4)} & =M \sum_{\langle i j\rangle}\left(\sigma_{i}^{y} \sigma_{j}^{y} \sigma_{k}^{y}-\sigma_{i}^{x} \sigma_{j}^{x} \sigma_{k}^{y}\right. \\
& \left.-\sigma_{i}^{x} \sigma_{j}^{y} \sigma_{k}^{x}-\sigma_{i}^{y} \sigma_{j}^{x} \sigma_{k}^{x}\right),
\end{aligned}
$$

where $M \sim J^{4} / K^{3}$

The main part of the effective Hamiltonian (2) describes the effective spin $\sigma=1 / 2 X Y$ model which has in two and three dimensions a "magnetic" $\langle\sigma\rangle \neq 0$ long range order in the ground state. Of course, the small symmetry breaking perturbations (3) should not destroyed the long range order. On the contrary we would expect that this perturbation should stabilize the long range ground state order also in one dimension model which is critical without symmetry breaking term. The question is what kind of order in the original $S=2$ model implies the "magnetic" order in the effective $\sigma=1 / 2$ model. In rather crude approximation we have found that in $d=2$ and 3 this "magnetic" order in effective model leads to quadrupolar long range order in the original model and in the second order calculations the quadrupolar order parameters are given by

$$
\left\langle\left(S_{i}^{x}\right)^{2}-2\right\rangle=2-\frac{z}{8}\left(\frac{J}{K}\right)^{2},
$$




$$
\left\langle\left(S_{i}^{y}\right)^{2}-2\right\rangle=\left\langle\left(S_{i}^{z}\right)^{2}-2\right\rangle=1+\frac{z}{16}\left(\frac{J}{K}\right)^{2},
$$

( $x, y$, and $z$ can be of course interchanged).

As mentioned above in $d=1$ the model described by the main part of the effective Hamiltonian is critical and to prove the existence of the long range order in this case one should take into account the higher order terms. However, to study the ground state behaviour of the cubic ferromagnetic chain one can use much more reliable and efficient method - DMRG, which can be applied directly to the original $S=2$ model [8]. Before we present the result of the DMRG method for the ground state we propose the RSRG technique to study the effective $\sigma=1 / 2$ one dimensional model which seems to be interesting by itself.

\section{LINEAR RSRG TRANSFORMATION}

It is well known that for the one dimensional Ising model the linear RSRG transformation - the decimation can be carried out exactly. In the quantum case, because of the non-commutativity of several terms of the Hamiltonian it is impossible to carry out the decimation exactly even in one dimension. However, Suzuki and Takano [13] proposed some approximation which leads to reasonable values for the free energy of the one dimensional quantum anisotropic Heisenberg model, especially in the higher temperature. In this paper we propose the linear RSRG transformation which can be used to study more general models, for example with three spin interaction. Additionally this method leads to better results for the free energy than those found in [13].

Below in this section, we study one dimensional system described by the Hamiltonian found in the previous section

$$
\begin{aligned}
H & =k_{x y} \sum_{i=0}^{N-1}\left(\sigma_{i}^{x} \sigma_{i+1}^{x}+\sigma_{i}^{y} \sigma_{i+1}^{y}\right)+k_{z} \sum_{i=0}^{N-1} \sigma_{i}^{z} \sigma_{i+1}^{z} \\
& +m \sum_{i=0}^{N-2}\left(\sigma_{i}^{y} \sigma_{i+1}^{y} \sigma_{i+2}^{y}-\sigma_{i}^{x} \sigma_{i+1}^{x} \sigma_{i+2}^{y}\right. \\
& \left.-\sigma_{i}^{x} \sigma_{i+1}^{y} \sigma_{i+2}^{x}-\sigma_{i}^{y} \sigma_{i+1}^{x} \sigma_{i+2}^{x}\right)
\end{aligned}
$$

where $i$ denotes the lattice site of the chain with $N+1$ sites and $-\beta=-1 / k T$ has already been absorbed into the Hamiltonian. For $k_{z}=0$ and $m=0$ the Hamiltonian (6) reduces to $X Y$ chain, exactly solved by Katsura [12].

We consider the linear RSRG transformation with scale factor $l=2$ :

$$
\begin{aligned}
\exp \left[G+H^{\prime}(\mathbf{s})\right] & =\operatorname{Tr} \boldsymbol{\sigma} \prod_{i=0}^{N / 2-1} \frac{1}{2^{N / 2}}\left(1+\mathbf{s}_{2 i+1} \cdot \boldsymbol{\sigma}_{2 i+1}\right) \\
& \times \exp [H(\boldsymbol{\sigma})]
\end{aligned}
$$

We divide the Hamiltonian (6) into six spin clusters

$$
H=\sum_{n=0}^{N / 5-1} \sum_{i=0}^{4} H\left(\boldsymbol{\sigma}_{n+i}, \boldsymbol{\sigma}_{n+i+1}, \boldsymbol{\sigma}_{n+i+2}\right)
$$

(six is the lowest number of spins which allows to reproduce the Hamiltonian (6) in the RSRG procedure).

By considering only one cluster, the renormalization can be carried out as follows

$$
\begin{aligned}
& \exp \left[G+H^{\prime}\left(\mathbf{s}_{1}, \mathbf{s}_{3}, \mathbf{s}_{5}\right)\right]=\frac{1}{8} \operatorname{Tr} \boldsymbol{\sigma} \prod_{k=1}^{3}\left(1+\mathbf{s}_{k} \cdot \boldsymbol{\sigma}_{k}\right) \\
& \times \exp \left[\sum_{i=0}^{4} H\left(\boldsymbol{\sigma}_{i}, \boldsymbol{\sigma}_{i+1}, \boldsymbol{\sigma}_{i+2}\right)\right] .
\end{aligned}
$$

This transformation takes quantum effect into account within a single cluster. However, contrary to the decimation procedure [13] it does not become exact even if the single cluster Hamiltonian commutes with each other. To get correct results for the renormalized coupling of the Ising model we have considered six spins of the given cluster on a ring. It is easy to check that such a procedure leads to the exact results. The results for the free energy of the one-dimensional $X Y$ model found by using the transformation (9) are shown in Tabl. 1 in comparison with the exact results [12] and the results found by using Suzuki-Takano decimation procedure [13].

\begin{tabular}{|l|l|l|l|}
\hline \multicolumn{1}{|c|}{$k_{x y}$} & \multicolumn{1}{|c|}{$F_{S T}$} & \multicolumn{1}{|c|}{$F$ (this paper) } & $F_{e x}$ \\
\hline \hline 0.1 & 0.703114 & 0.703098 & 0.703098 \\
\hline 0.2 & 0.732625 & 0.732376 & 0.732374 \\
\hline 0.3 & 0.780567 & 0.779415 & 0.779393 \\
\hline 0.4 & 0.845252 & 0.842049 & 0.841906 \\
\hline 0.5 & 0.924621 & 0.917933 & 0.917409 \\
\hline 0.6 & 1.016454 & 1.004827 & 1.003466 \\
\hline 0.7 & 1.118576 & 1.100726 & 1.097914 \\
\hline 1.5 & 2.1233 & 2.0371 & 2.0008 \\
\hline 3.0 & 4.2087 & 3.9902 & 3.8637 \\
\hline 5.0 & 7.0257 & 6.6442 & 6.39245 \\
\hline
\end{tabular}

Table 1. Approximate free energies obtained by Takano and Suzuki $\left(F_{S T}\right)$, in this paper $(F)$ and the exact free energy obtained by Katsura $\left(F_{e x}\right)$ for the one dimensional spin-1/2XY model.

From the results shown in Table 1 , one can see that $F$ found by using the transformation (9) in this paper is a better approximation to $F_{e x}$ than $F_{S T}$ found in [13] and for relatively low temperature $k_{x y}=5$ it leads to a reasonable value which differs less than by 
$1 \%$ from the exact result. It is interesting to note that even the value of the ground state internal energy per spin of the $X Y$ model found by using transformation (9) $E_{0}=-4 / 3$ is in a good agreement with the exact result $E_{0}=-4 / \pi \approx-1.2732$, however, in principle, the approximation used in this paper is of course a hightemperature approximation.

Applying the transformation (9) to the Hamiltonian (6) , we obtain the transformed Hamiltonian $H^{\prime}$ in the same form as Eq. (6) for new spin operators (s) with new parameters $k_{x y}^{\prime}, k_{z}^{\prime}$ and $m^{\prime}$ :

$$
\begin{aligned}
k_{x y}^{\prime} & =\frac{1}{6}\left[\log \left(z_{0}+4 f_{x}-f_{z}\right)-\log \left(z_{0}-2 f_{x}\right)\right], \\
k_{z}^{\prime} & =\frac{1}{24}\left[3 \log \left(z_{0}+4 f_{m}+3 f_{z}\right)+3 \log \left(z_{0}+4 f_{m}+3 f_{z}\right)\right. \\
& \left.-4 \log \left(z_{0}-2 f_{x}-f_{z}\right)-2 \log \left(z_{0}+4 f_{x}-f_{z}\right)\right], \\
m^{\prime} & =\frac{1}{8}\left[\log \left(z_{0}+4 f_{x}+3 f_{z}\right)-\log \left(z_{0}-4 f_{m}+3 f_{z}\right)\right]
\end{aligned}
$$

where

$$
\begin{gathered}
f_{x}=\left\langle\sigma_{1}^{x} \sigma_{3}^{x}\right\rangle, \quad f_{z}=\left\langle\sigma_{1}^{z} \sigma_{2}^{z}\right\rangle, \\
f_{m}=\left\langle\sigma_{1}^{y} \sigma_{3}^{y} \sigma_{5}^{y}\right\rangle .
\end{gathered}
$$

The angular brackets denote a partial expectation value, defined for some operator $A$ by

$$
\langle A\rangle=\operatorname{Tr}_{\boldsymbol{\sigma}} A P \exp [H] \quad \text { and } \quad z_{0}=\operatorname{Tr} \boldsymbol{\sigma} P \exp [H]
$$

As usual in each step of the transformation a constant term independent of $s^{\alpha}$ appears

$$
\begin{aligned}
g_{0} & =\frac{1}{8}\left[4 \log \left(z_{0}-2 f_{x}-f_{z}\right)+2 \log \left(z_{0}+4 f_{x}-f_{z}\right)\right. \\
& \left.=\log \left(z_{0}-4 f_{m}+3 f_{z}\right)+\log \left(z_{0}-4 f_{m}-3 f_{z}\right)\right] .
\end{aligned}
$$

We can calculate the free energy $F$ per site of the system from the renormalization transformation by using the well known formula

$$
F=\frac{1}{3} \sum_{n=0}^{\infty} \frac{g_{0}^{(n)}}{2^{(n)}},
$$

where $n$ denotes $n$th step of the transformation.

In the Fig. 1 the free energy and internal energy of the model (6), for original $k_{z}=0$, as functions of $x=\arctan \left(\sqrt{m / k_{x y}}\right)$ are presented for temperatures $T=2,1 / 1.1$ and $1 / 1.5$ (in the exchange interaction units). In the case of the internal energy we also present the results for the very low temperature $T \rightarrow 0$. The parameter $x$ is chosen in such a way to have for arbi- trary values of $m$ and $k_{x}$ the ground state energy per spin equal 1 for the classical system. This allows us to compare the deviations from the classical ground state for several models. It is easy to see that the deviation depends on the ratio $m / k_{x y}$ and is maximal for $x=\pi / 2$, it means for the system with only three spin interactions. The value of the ground state energy of such a system $E / N \approx-2.31$ can be compared with the energy of the pure $X Y$ model $(m=0)$ found in our approximation, $E / N \approx-4 / 3$ (the exact value is $-4 / \pi[12]$ ) and with the value for the classical ground state $E / N=-1$.
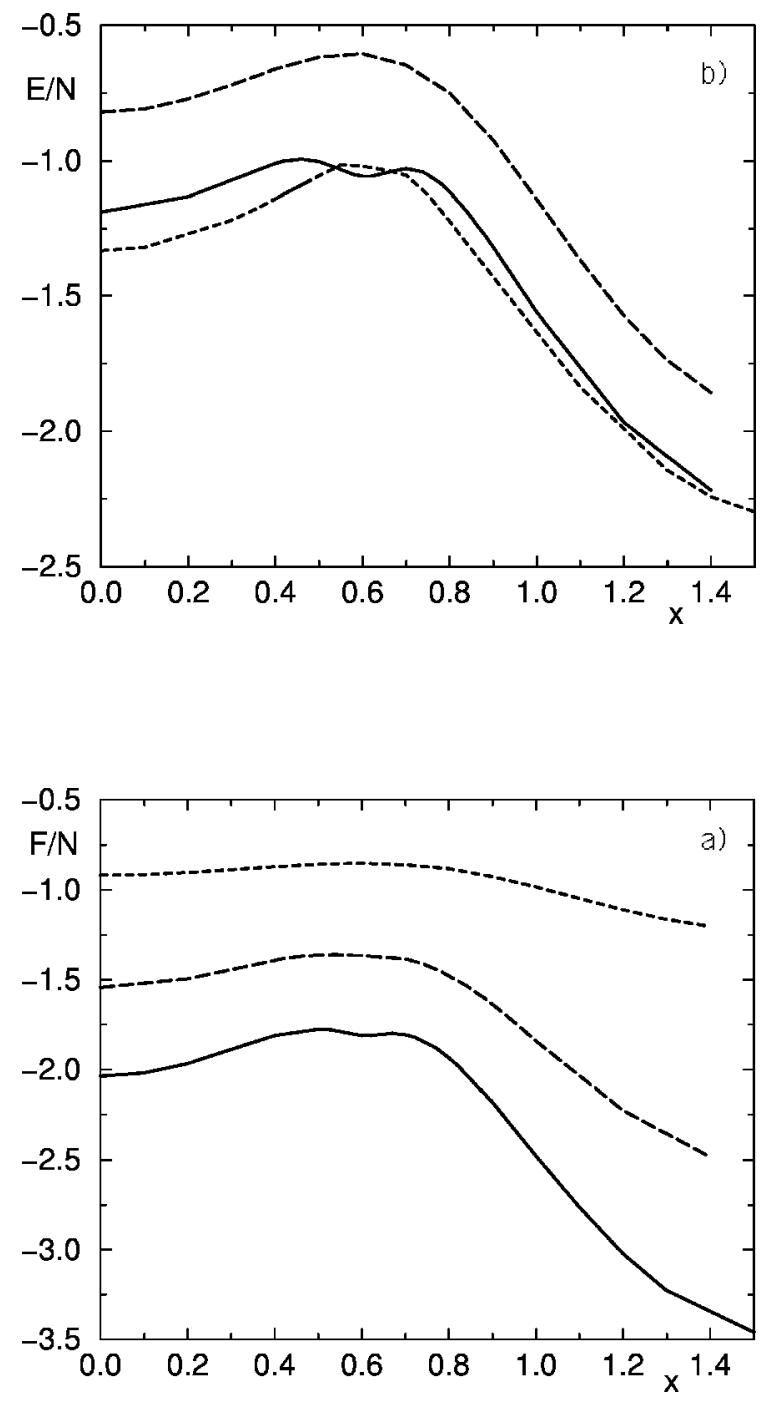

Fig. 1. a). The free energy of the model (5) as functions of parameter $x=\operatorname{arctanh}\left(\sqrt{m / k_{x y}}\right)$ for $k_{z}=0$ and $T=2$ (dotted line), 1/1.1 (dashed line), 1/1.5 (solid line). b). The internal energy of the model (5) as functions of parameter $x=\operatorname{arctanh}\left(\sqrt{m / k_{x y}}\right)$ for $k_{z}=0$ and $T=2$ (dashed line), 1/1.1 (solid line) and $T \rightarrow 0$ (dotted line).

In Fig. 2 the specific heat as a function of temperature for $k_{z}=0$ and (i) $m=0$, pure $X Y$ model; (ii) $k_{x}=0$, pure three spin interaction model and (iii) $m=0.2 k_{x}$, is shown. As one could expect our approximation is worse 
for the lower temperature which is seen in the Fig. $2 b$ where we compare our result for the $X Y$ model with the exact result found by Katsura [12]. Unfortunately, our approximation fails for higher values of three-spin interaction $(m)$ at low temperature and leads to unphysical result for the specific heat.
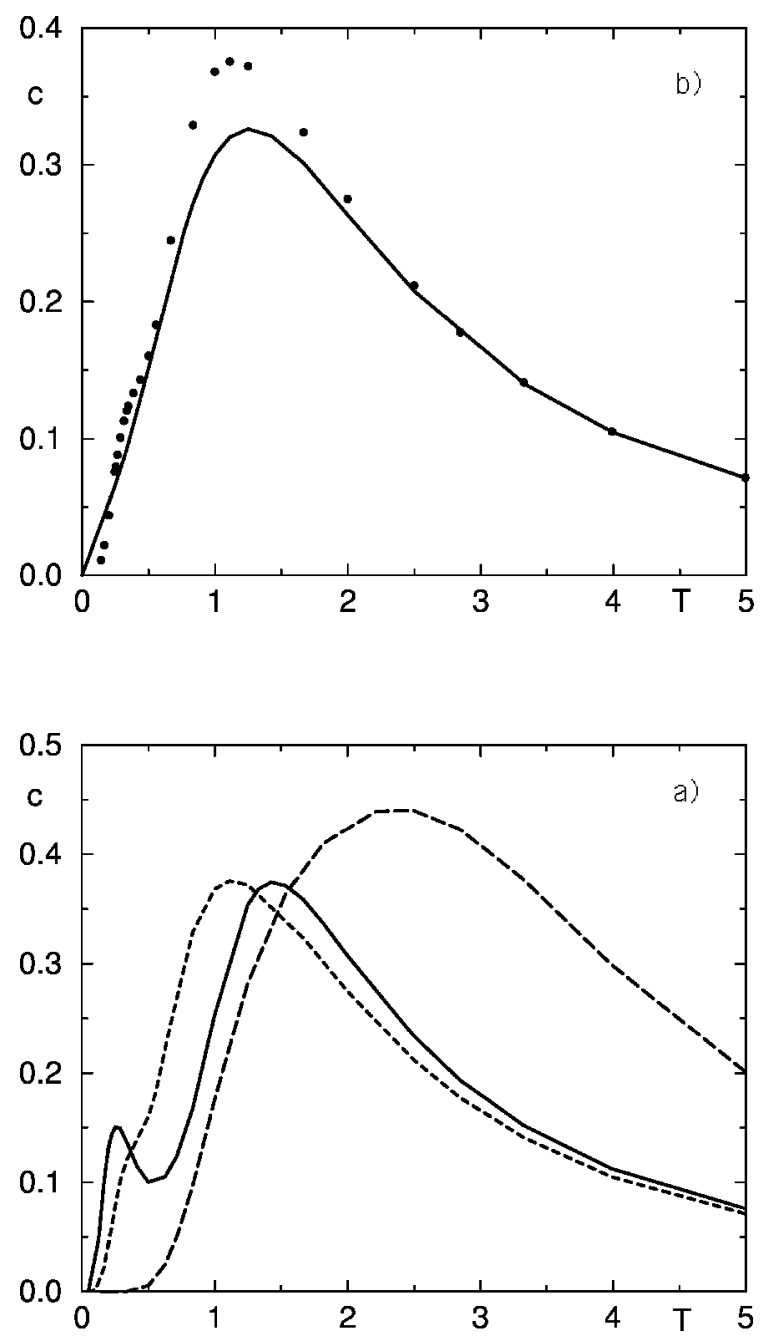

Fig. 2. a). The temperature dependence of the specific heat of the model (5) as function of temperature for $k_{z}=0$ and (i) $m=0$, pure $X Y$ model (dotted line), (ii) $k_{x}=0$, pure three spin interaction model (dashed line) and (iii) $m=0.2 k_{x}$ (solid line). b). The specific heat obtained in this paper (dots) and the exact result obtained by Katsura (solid line) for one-dimensional spin-1/2 XY model.

\section{DENSITY MATRIX RENORMALIZATION GROUP}

There is no fully reliable method which would enable us to investigate the original model (1) in two and three dimensions for general $K$. However, it is possible to confirm the existence of the purely quadrupolar phase driven by quantum fluctuations in the one dimensional system by using density matrix renormalization group. DMRG is one of the most reliable numerical techniques to study the ground state and low-lying excited states of $1 \mathrm{D}$ quantum lattice models [14].

In order to obtain the zero-temperature phase diagram and critical properties of the model (1) we carried out extensive numerical calculations using exact diagonalization techniques on short chain and the DMRG on longer system [8]. To check the existence of the magnetic and quadrupolar long range order we applied to the first and last spins of the $L$-spin open chain a symmetrybreaking auxiliary fields. Then the Hamiltonian which was simulated in the numerical calculation was

$$
\begin{array}{r}
H \rightarrow H-h_{m}^{\alpha}\left(S_{1}^{\alpha}+S_{L}^{\alpha}\right) \\
-h_{q}^{\alpha}\left[\left(S_{1}^{\alpha}\right)^{2}+\left(S_{L}^{\alpha}\right)^{2}\right] .
\end{array}
$$

Using the Hamiltonian (15) we were able to investigate the spontaneous magnetization $m^{\alpha}=\left\langle S^{\alpha}\right\rangle$ and quadrupolar order parameter $q^{\alpha}=\left\langle\left(S^{\alpha}\right)^{2}-2\right\rangle$, and corresponding correlation lengths.

Evidence has been obtained that, in qualitative agreement with the MFA prediction, the ground state magnetization diminishes continuously when $K \rightarrow K_{c}$. In the best approximation we found that $K_{c} \approx-3.71 J$ which can be compared with the MFA value $K_{c}=-8 J$. We also found the critical indices describing the critical behaviour of the magnetization and the correlation length

$$
\begin{gathered}
m(k) \infty\left(K_{c}-K\right)^{\beta}, \quad \beta=0.127 \pm 0.004 ; \\
\xi_{m} \infty\left(K-K_{c}\right)^{-\nu}, \quad \nu=1.02 \pm 0.06 .
\end{gathered}
$$

It is worth noting that the values of both the exponents $\beta, \nu$ are close to those of the two-dimensional Ising model. So, at $K=K_{c}$ our system undergoes the continuous phase transition from magnetic to nonmagnetic phase and this phase transition belongs to the 2D Ising model universality class.

In contrary to the MFA predictions we found both in the magnetic and nonmagnetic phases the long range quadrupolar order. We were able to analyze the dependence of $q$ on $K$ close to the magnetic phase transition point $K_{c}$. For $K>K_{c}$ we assume a power-law singularity and a linear term

$$
q(K)=q_{\mathrm{left}}+c_{1}\left(K_{c}-K\right)^{\theta}+c_{2}\left(K_{c}-K\right)
$$

Fitting yields $q_{\text {left }}=1.838 \pm 0.01, \theta=0.5 \pm 0.1$. Thus, on this side of the phase transition point, the quadrupolar order parameter appears to have singular behaviour, but the exponent $\theta$ differs from $\beta$ describing the magnetization. For $K<K_{c}$, results suggest the regular behaviour and our fitting formula is

$$
q(K)=q_{\mathrm{right}}+c_{3}\left(K_{c}-K\right)+c_{4}\left(K-K_{c}\right)^{2} .
$$

and $q_{\text {right }}=1.840 \pm 0.002$. The left and right estimates of 
the order parameter $q$ at $K=K_{c}$ are in good agreement, which supports our scaling assumptions. For correlation length corresponding to the quadrupolar order parameter $\left(\xi_{q}\right)$ our fitting yields rather rough estimates, the errors are believed to be around $20 \%$. The results suggest that $\xi_{q}=\xi_{m}$ for $\mathrm{K}>K_{c}$, which is expected answer, since the magnetic and quadrupolar order is intimately connected in this phase. For $K<K_{c}$, the correlation length at first decreases but starting with $K \approx-3.9$ increases. Due to the poor precision we cannot resolve whether $\xi_{q}$ diverges for $K \rightarrow K_{c}$ or it remains finite. The confirmation of the purely quadrupolar phase in the one dimensional case give rise to the belief that such a phase should also exist in higher dimensions at sufficiently low temperatures if the cubic crystal field is strong. Increasing temperature should destroy the quadrupolar long range order and leads to a finite temperature phase transition between the quadrupolar and completely disordered phase. Note that the correct theory of this transition cannot be obtained by simply substituting spins with classical vectors in our model, since this approach is unable to account for the purely quadrupolar order. The usual practice of neglecting quantum fluctuation by treating spins classically around a finite-temperature phase transition would confront fundamental difficulties in this case. As mentioned above the correct description of this phase transition cannot be also found by using any kind of the MFA. In the next section we will present how this latter problem can be omitted by using simple transformation similar to the linear RSRG transformation.

\section{MEAN FIELD APPROXIMATION TO RENORMALIZED HAMILTONIAN}

Below in this Section we will present method which can be used to study the $3 \mathrm{D}$ cubic model at finite temperature. Usually, the MFA is believed to provide qualitatively correct description of phase diagrams of three dimensional spin models. However, this method is unable to account for the purely quadrupolar long range order when applied directly to the Hamiltonian (1). Therefore, we will first renormalize the Hamiltonian (1) to get rid of the crystal field term. This will lead to some renormalized Hamiltonian which will be treated using the MFA [7].

The renormalized Hamiltonian $H_{r e n}$ is defined by the operator equation

$$
\exp \left[H_{\mathrm{ren}}(\boldsymbol{\sigma})\right]=\operatorname{Tr}_{\mathbf{S}}(\boldsymbol{\sigma}, \mathbf{S}) \exp [H(\mathbf{S})]
$$

with the projection operator $P(\sigma, S)$ which couples the original spin $(S)$, and effective spin $(\sigma)$ space and due to the translational invariance, one can assume

$$
P\left(\mathbf{S}^{\prime}, \mathbf{S}\right)=\prod_{i} p\left(\mathbf{S}_{i}^{\prime}, \mathbf{S}_{i}\right)
$$

where the product is taken over all lattice sites. The most general rotationally invariant projector for the spin2 model is

$$
p\left(\boldsymbol{\sigma}_{i}, \mathbf{S}_{i}\right)=\sum_{n=0}^{4} a_{n}\left(\boldsymbol{\sigma}_{i} \cdot \mathbf{S}_{i}\right)^{n}
$$

However, we can confine ourselves to the simpler form

$$
p\left(\boldsymbol{\sigma}_{i}, \mathbf{S}_{i}\right)=a_{0}+a_{1}\left(p\left(\boldsymbol{\sigma} \cdot \mathbf{S}_{i}\right)+a_{2} p\left(\boldsymbol{\sigma} \cdot \mathbf{S}_{i}\right)^{2}\right.
$$

which is sufficient to find the appropriate renormalized Hamiltonian.

The projection operator $P(\sigma, S)$ must satisfy the condition

$$
\operatorname{Tr} \boldsymbol{\sigma} p(\boldsymbol{\sigma}, \mathbf{S})=1
$$

which insures that the partition function of the original and effective spin problem are the same, and

$$
\operatorname{Tr}_{\mathbf{S}} S^{\alpha} p=\sigma^{\alpha}, \quad \operatorname{Tr}_{\mathbf{S}}\left(S^{\alpha}\right)^{2} p=\left(\sigma^{\alpha}\right)^{2}
$$

which insures that equation (18) transforms the original system in itself for $K=0$ (isotropic case). It is easy to see that the conditions (22) and (23) are fulfilled if $a_{0}=-13 / 35, a_{1}=13 / 105$ and $a_{2}=1 / 21$.

In order to solve equation (18) one has to use some approximation, for example the cumulant expansion. In the first order calculation the effective Hamiltonian contains only spin-spin interaction between the nearest neighbours where

$$
\begin{aligned}
& H_{\mathrm{ren}}^{(1)}=-J \sum_{\langle i, j\rangle}\left(\boldsymbol{\sigma}_{i} \cdot \boldsymbol{\sigma}\right), \\
& J_{p}=\frac{8 \kappa e^{-2 \kappa}-2 e^{-4 \kappa}+\kappa+6}{\kappa\left(3+2 e^{-2 \kappa}\right)^{2}},
\end{aligned}
$$

and $\kappa=\beta K$.

In the second-order approximation for the cubic lattice there come into play nine interactions with six different coupling parameters. Between others, due to the interaction of the spins with the crystal field, the effective quadrupole-quadrupole interactions $\left(J_{3}, J_{4}\right)$ appear

$$
-J_{3} \sum_{\langle i, j\rangle, \alpha}\left(\sigma_{i}^{\alpha}\right)^{2}\left(\sigma_{j}^{\alpha}\right)^{2}-J_{4} \sum_{\langle i, j\rangle, \alpha, \beta} \sigma_{i}^{\alpha} \sigma_{i}^{\beta} \alpha_{j}^{\alpha} \sigma_{j}^{\beta}
$$

These interactions can of course cause the existence of the quadrupolar ordering even in the non-magnetic phase. The renormalized Hamiltonian can be used as a starting point for MFA to analyze the possibility of the phase transition to the magnetic phase with order parameter $m^{\alpha}$ and to the quadrupolar phase with the order parameter $q^{\alpha}$. It has been shown that the phase diagram 
of the cubic ferromagnet with three easy axes consists three lines which describe the continuous phase transition to the magnetic phase for $K>K_{t} \approx-1.94\left(K_{t}\right.$ denotes tricritical point), the discontinuous phase transition to the magnetic phase for $-1.94>K>-7.6$ and the discontinuous phase transition to the purely quadrupolar phase for $K<-7.6$.

\section{CONCLUSIONS}

The purpose of this paper lies in showing that there are spin systems for which the quantum fluctuations cannot be neglected even in the vicinity of the finite temperature transition point. This conclusion is valid for all dimensionality and for values of the spin $S \geq 2$. It has been proved by using several methods that in opposite to the MFA results there is the finite temperature phase transition from paramagnetic to quadrupolar phase in three-axial cubic ferromagnets in 2 and 3 dimensions for sufficiently large, negative, anisotropy con- stant. The ground state of the ferromagnet with the cubic single-ion anisotropy is ordered for an arbitrary value of the anisotropy $K$, and for all dimensions. However, for $K>K_{c}$ one observes the magnetic long range order whereas for $K<K_{c}$ the quadrupolar long range order. $K_{c}$ is negative and its value depends on the space dimensionality. The existence of the quadrupolar order in the non-magnetic state of the system without quadrupolar interactions is a pure quantum effect which could not be observed in the case of the classical spins. We have also applied the linear RSRG transformation to study the temperature dependence of the energy of the one dimensional cubic ferromagnet described by the effective Hamiltonian with bilinear $\left(k_{x y}\right)$ and three spin interactions $(m)$. It has been shown that in the model with cubic symmetry the deviation of the ground state from the classical ground state is much larger than in the pure XY model.

This paper was partially supported (M.D.) by the State Committee for Scientific Research, Grant No. 2P03B07214.
[1] F. D. M. Haldane, Phys. Lett. A 93, 464(1983).

[2] A. Klümper, A. Schadschneider, J. Zittartz, Z. Phys. B 87, 281 (1992); H. Niggemann, J. Zittartz, Z. Phys B 101, 289 (1996); H. Niggemann, A. Klümper and J. Zittartz, Z. Phys. B 104, 103 (1996).

[3] J. Sznajd, J. Magn. Magn. Mat. 42, 269 (1984).

[4] V.V. Valkov, T.A. Valkova, Teor. Mat. Fiz. 59, 269 (1984).

[5] Z. Domański, J. Sznajd, Phys. Stat. Sol. B 129, 135 (1985).

[6] M. Dudziński, J. Sznajd, Eur. Phys. J. B 5, 745 (1998).

[7] J. Sznajd, M. Dudziński, Phys. Rev. B59, 4176(1999).
[8] M. Dudziński, G. Fath, J. Sznajd, Phys. Rev. B 59, 13764 (1999).

[9] A. Aharony, Phys. Rev. B 8, 4270 (1973).

[10] H. Kleinert, S. Thoms, V. Schulte-Frohlinde, Phys. Rev. B 56, 14428 (1997).

[11] T. Kennedy, E. H. Lieb, B. Siriam Shastry, Phys. Rev. Lett. 61, 2582 (1988).

[12] S. Katsura, Phys. Rev. 127, 1508 (1962).

[13] M. Suzuki, H. Takano, Phys. Lett. A 69, 426 (1979); H. Takano, M. Suzuki, J. Stat. Phys. 26, 635 (1981).

[14] S. R. White, Phys. Rev. Lett. 69, 2863 (1992); Phys. Rev. B 48, 10345 (1993).

\title{
КВАНТОВІ ФЛЮКТУАЩЇ В КУБІЧНИХ ФЕРОМАГНЕТИКАХ
}

\author{
Ю. ШШнайд, М. Дудзіньскі \\ Інститут низъких температур та структурних досліджень Польсъкой академіӥ наук, \\ 50-950, Вроияав, Польща \\ E-mail: sznajd@apollo.int.pan.wroc.pl
}

\begin{abstract}
Зроблено огляд теорії фазових переходів у квантових спінових феромагнетиках з кубічною однойонною анізотропією. Обговорено деякі основні труднощі, що виникають при середньопольовому підході до цих систем. Звернуто увагу на те, що квантові флюктуащії викликають квадрупольне далекосяжне впорялккування у кубічних феромагнетиках з трьома легкими осями. Џе впорядкування не спостерігається в класичному варіянті аналізованої моделі. Для вивчення магнетних ланцюжків з багатоспіновою взаємодією запропоновано нову процедуру, децимапії. Ї̈ застосовано при дослідженні ефективного гамільтоніяна кубічного феромагнетика з сильною анізотропією.
\end{abstract}

